\title{
U.S. Hydropower Resource Assessment for Hawaii
}

\author{
Prepared by: \\ James E. Francfort \\ Project Manager: \\ Ben N. Rinehart
}

Published September 1996

Idaho National Engineering Laboratory Renewable Energy Products Department Lockheed Martin Idaho Technologies Company Idaho Falls, Idaho 83415

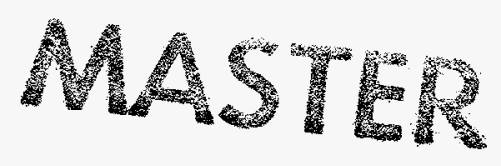

Prepared for the

U.S. Department of Energy

Assistant Secretary for Energy Efficiency and Renewable Energy

Under DOE Idaho Operations Office

Contract DE-AC07-94ID13223 


\section{DISCLAIMER}

This report was prepared as an account of work sponsored by an agency of the United States Government. Neither the United States Government nor any agency thereof, nor any of their employees, makes any warranty, express or implied, or assumes any legal liability or responsibility for the accuracy, completeness, or usefulness of any information, apparatus, product, or process disclosed, or represents that its use would not infringe privately owned rights. Reference herein to any specific commercial product, process, or service by trade name, trademark, manufacturer, or otherwise does not necessarily constitute or imply its endorsement, recommendation, or favoring by the United States Government or any agency thereof. The views and opinions of authors expressed herein do not necessarily state or reflect those of the United States Government or any agency thereof. 


\section{DISCLAIMER}

Portions of this document may be illegible in electronic image products. Images are produced from the best available original document. 


\begin{abstract}
The U.S. Department of Energy is developing an estimate of the undeveloped hydropower potential in the United States. The Hydropower Evaluation Software (HES) is a computer model that was developed by the Idaho National Engineering Laboratory for this purpose. HES measures the undeveloped hydropower resources available in the United States, using uniform criteria for measurement. The software was developed and tested using hydropower information and data provided by the Southwestern Power Administration. It is a menu-driven program that allows the personal computer user to assign environmental attributes to potential hydropower sites, calculate development suitability factors for each site based on the environmental attributes present, and generate reports based on these suitability factors. This report describes the resource assessment results for the State of Hawaii.
\end{abstract}




\section{CONTENTS}

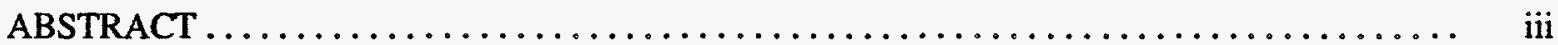

ACKNOWLEDGMENTS $\ldots \ldots \ldots \ldots \ldots \ldots \ldots \ldots \ldots \ldots \ldots \ldots \ldots \ldots \ldots \ldots \ldots \ldots \ldots \ldots \ldots \ldots$

INTRODUCTION $\ldots \ldots \ldots \ldots \ldots \ldots \ldots \ldots \ldots \ldots \ldots \ldots \ldots \ldots \ldots \ldots \ldots \ldots \ldots \ldots \ldots \ldots \ldots$

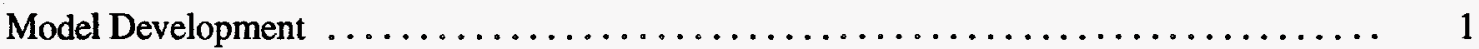

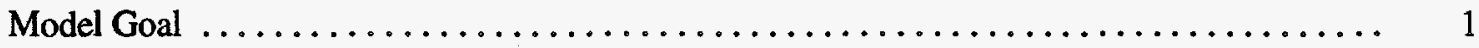

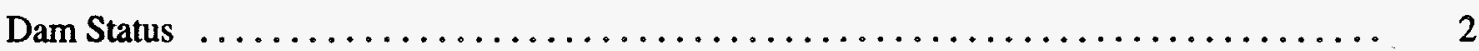

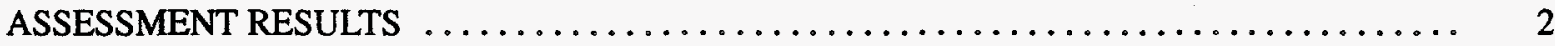

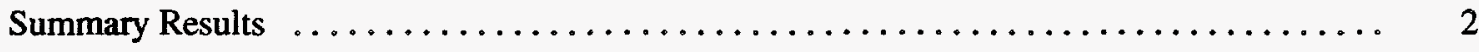

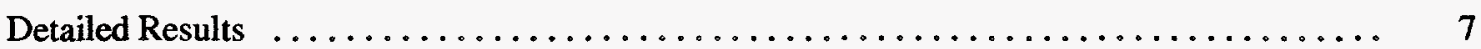

OBTAINING INDIVIDUAL STATE INFORMATION $\ldots \ldots \ldots \ldots \ldots \ldots \ldots \ldots \ldots \ldots \ldots$

ADDITIONAL HYDROPOWER EVALUATION SOFTWARE INFORMATION . . . . . . . . 9

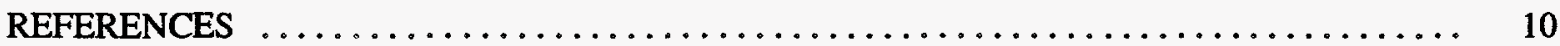

APPENDIX A-Summary Report $\ldots \ldots \ldots \ldots \ldots \ldots \ldots \ldots \ldots \ldots \ldots \ldots \ldots \ldots \ldots \ldots \ldots \ldots \ldots \ldots \ldots \ldots$

APPENDIX B-Hawaii Sites Listing $\ldots \ldots \ldots \ldots \ldots \ldots \ldots \ldots \ldots \ldots \ldots \ldots \ldots \ldots \ldots \ldots \ldots \ldots \ldots$

APPENDIX C_Individual Resource Database Listing $\ldots \ldots \ldots \ldots \ldots \ldots \ldots \ldots \ldots \ldots \ldots \ldots \ldots$

APPENDIX D-List of 19 Additional Hydropower Sites $\ldots \ldots \ldots \ldots \ldots \ldots \ldots \ldots \ldots$ D-1

\section{FIGURES}

1. Number of sites, by capacity groups, with HES-modeled undeveloped

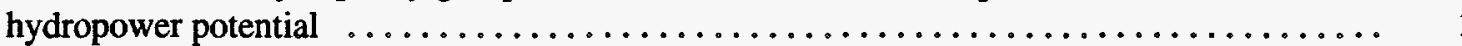

2. The nonmodeled and HES-modeled undeveloped hydropower potential $\ldots \ldots \ldots \ldots \ldots$

3. The number of sites with undeveloped hydropower potential and the total megawatts of HES-modeled undeveloped hydropower potential ......................

4. The Wainiha Hydroelectric Plant, on the Island of Kauai, is an example of a developed site in

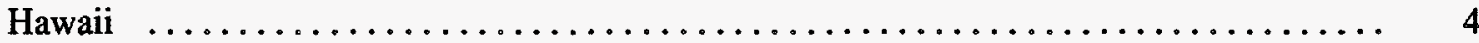

5. Number of sites with undeveloped hydropower potential in the Hawaii Islands $\ldots \ldots \ldots \ldots$

6. Megawatts of HES-modeled undeveloped hydropower potential in the Hawaii Islands ..... 5

\section{TABLES}

1. Undeveloped hydropower potential summaries for Hawaii 


\section{ACKNOWLEDGMENTS}

The authors thank Peggy A. M. Brookshier and John V. Flynn of the U.S. Department of Energy, and David Rezachek of the State of Hawaii for their active participation and timely comments. 


\section{U.S. Hydropower Resource Assessment for Hawaii}

\section{INTRODUCTION}

In June 1989, the U.S. Department of Energy initiated the development of a National Energy Strategy to identify the energy resources available to support the expanding demand for energy in the United States. Public hearings conducted as part of the strategy development process indicated that undeveloped hydropower resources were not well defined. As a result, the Department of Energy established an interagency Hydropower Resource Assessment Team to ascertain the undeveloped hydropower potential. In connection with these efforts by the Department of Energy, the Idaho National Engineering Laboratory designed the Hydropower Evaluation Software (HES), which has been used to perform a resource assessment of the undeveloped conventional hydropower potential in over 30 states. This report presents the results of the hydropower resource assessment for the State of Hawaii. Undeveloped pumped storage hydropower potential is not included.

The HES was developed as a tool to measure undeveloped hydropower potential regionally or by state. The software is not intended to provide precise development factors for individual sites, but to provide regional or state totals. Because the software was developed as a generic measurement tool encompassing national issues, regional and state totals must be considered judiciously; various local issues may skew undeveloped hydropower potential totals. The information for the resource assessment was compiled from the Federal Energy Regulatory Commission's Hydroelectric Power Resources Assessment database and several other sources. Refer to DOE/ID-10338, the User's Manual (Francfort, Matthews, Rinehart 1991) for the specifics of the software and to DOE/ID-10430.1, the Status Report (Conner, Francfort, Rinehart 1996) for an overview of all resource assessment activities to date.

\section{Model Development}

Hydropower Evaluation Software, both a probability-factor computer model and a database, is a menu-driven program that is intended to be userfriendly. Computer screens and report-generation capabilities were developed to meet the needs of users nationwide. The software uses environmental attribute data for each potential site to generate an overall project environmental suitability factor (PESF) between 0.1 and 0.9 . The PESF reflects the considerations that (a) environmental concerns can make a potential site unacceptable, prohibiting its development (for a suitability factor of 0.1 ), or (b) if there are no environmental concerns, there is a higher likelihood of site development ( 0.9 indicating the highest likelihood of development). A combination of attributes can result in a lower suitability factor because multiple environmental considerations would reduce the likelihood that a site may be developed to its physical potential.

\section{Model Goal}

The goal of the HES is to assemble an accurate resource database of all sites with undeveloped hydropower potential in the United States for use as a planning tool to determine the viable national hydropower potential. Undeveloped hydropower potential is not limited to the development of new sites; it also includes the development of additional hydropower generating capacity at sites that currently have hydropower, but are not developed to their full potential. This undeveloped hydropower potential is a source of nonpolluting, renewable energy available to meet the growing power needs of the United States. The HES should help make this goal obtainable and ensure a set of uniform criteria for national assessment. 


\section{Dam Status}

The effects of environmental attributes vary by dam status. The dam status classifications used are as follows

$\mathrm{W}=$ Developed hydropower site with current power generation, but the total hydropower potential has not been fully developed. Only the undeveloped hydropower potential is discussed in this report.

$\mathrm{W} / \mathrm{O}=$ Developed site without current power generation. The site has some type of developed impoundment or diversion structure, but no developed hydropower generating capability.

$\mathrm{U}=$ Undeveloped site. The site does not have power generation capability nor a developed impoundment or diversion structure.

\section{ASSESSMENT RESULTS}

\section{Summary Results}

A total of 25 sites (Table 1) have been identified and assessed for their undeveloped hydropower potential. The HES results for individual site capacities range from 73 kilowatts $(\mathrm{kW})$ to 16 megawatts (MW). Most of the HES-modeled potential sites in Hawaii are small hydropower sites; only two individual sites are larger than $10 \mathrm{MW}$ (Figure 1).

The nonmodeled undeveloped hydropower potential total for Hawaii was identified as $428 \mathrm{MW}$. The HES results lowers this estimate about $84 \%$ to $67 \mathrm{MW}$. The greatest reduction in undeveloped hydropower potential, by MW, occurs at sites with no structures in place (undeveloped category). These sites have an HES-modeled undeveloped hydropower potential of $52 \mathrm{MW}$, a $354 \mathrm{MW}$ reduction in the estimated undeveloped hydropower potential (Figure 2). The developed sites, with power, have the lowest percentage decrease in modeled undeveloped potential capacity. The unadjusted potential is $2.9 \mathrm{MW}$, and the modeled capacity is $2.6 \mathrm{MW}$, a $10 \%$ decrease in capacity (Figure 2). As can be deducted from Figure 3, the developed sites without power have the smallest HES-modeled average capacity per site $(1.8 \mathrm{MW})$, and the undeveloped sites have the highest HES-modeled average capacity per site (3.0 MW). Figure 4 illustrates a developed site on the island of Kauai.

The potential sites are usually discussed and summed by river basins for most state reports. However, there are no large (by contiguous state standards) river basins in Hawaii, so the sites are identified for each of the individual Islands. The 25 identified sites are located on 4 of the Islands. The number of sites per Island range from 3 on Honolulu, to 9 on Kauai (Figure 5). Honolulu has the most undeveloped hydropower potential $(35 \mathrm{MW}$ ) of the 4 Hawaii Islands (Figure 6).

Table 1. Undeveloped hydropower potential summaries for Hawaii. The table contains the nonmodeled undeveloped nameplate potential and the HES-modeled undeveloped hydropower potential totals.

\begin{tabular}{lccc}
\hline & Number of projects & $\begin{array}{c}\text { Nameplate potential } \\
(\mathrm{MW})\end{array}$ & $\begin{array}{c}\text { HES-modeled potential } \\
\text { (MW) }\end{array}$ \\
\cline { 2 - 4 } With Power & 1 & 2.9 & 2.6 \\
W/O Power & 7 & 19.6 & 12.8 \\
Undeveloped & 17 & 405.6 & 51.6 \\
State Total & 25 & 428.1 & 67.0 \\
\hline
\end{tabular}




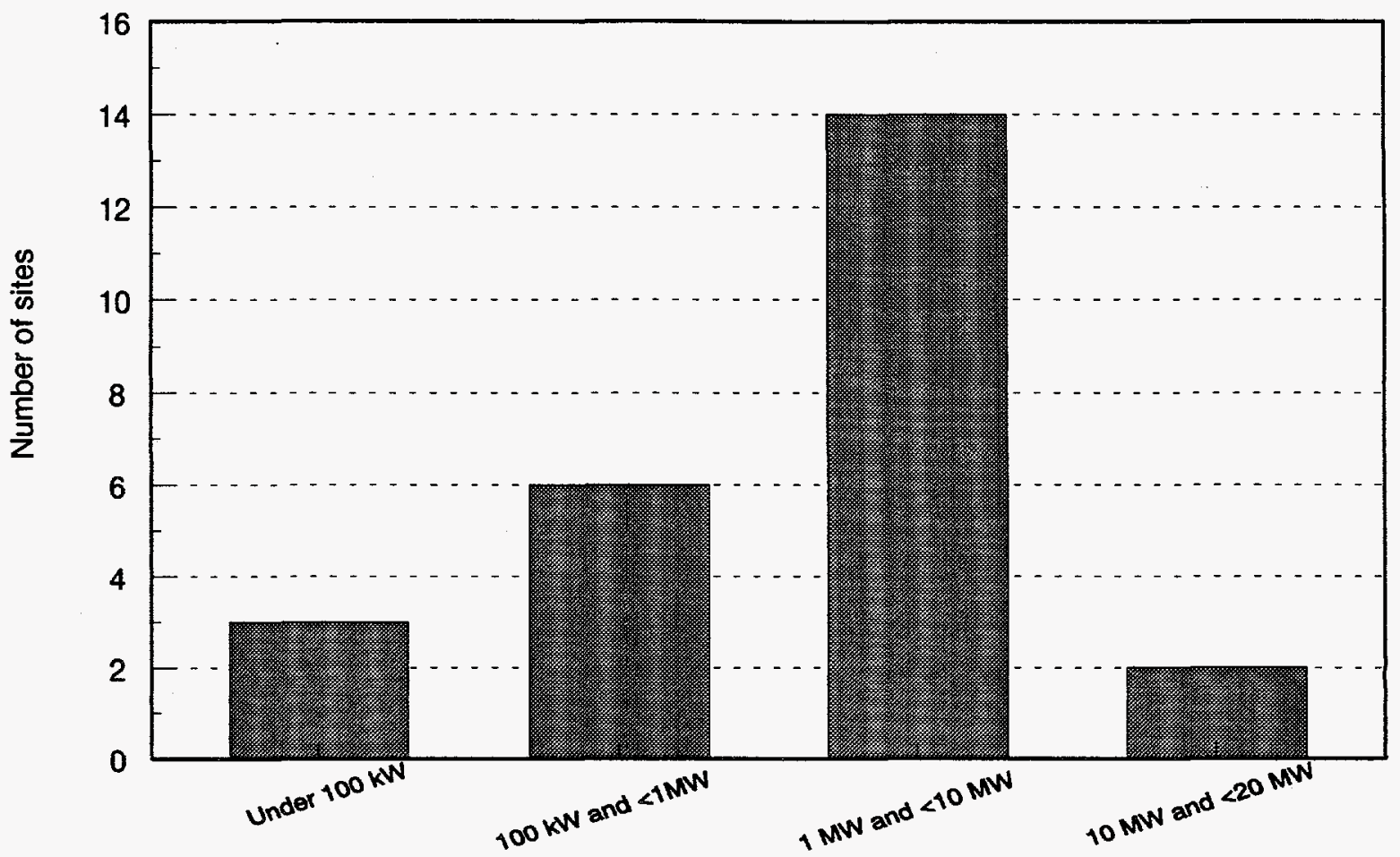

Figure 1. Number of sites, by capacity groups, with HES-modeled undeveloped hydropower potential.

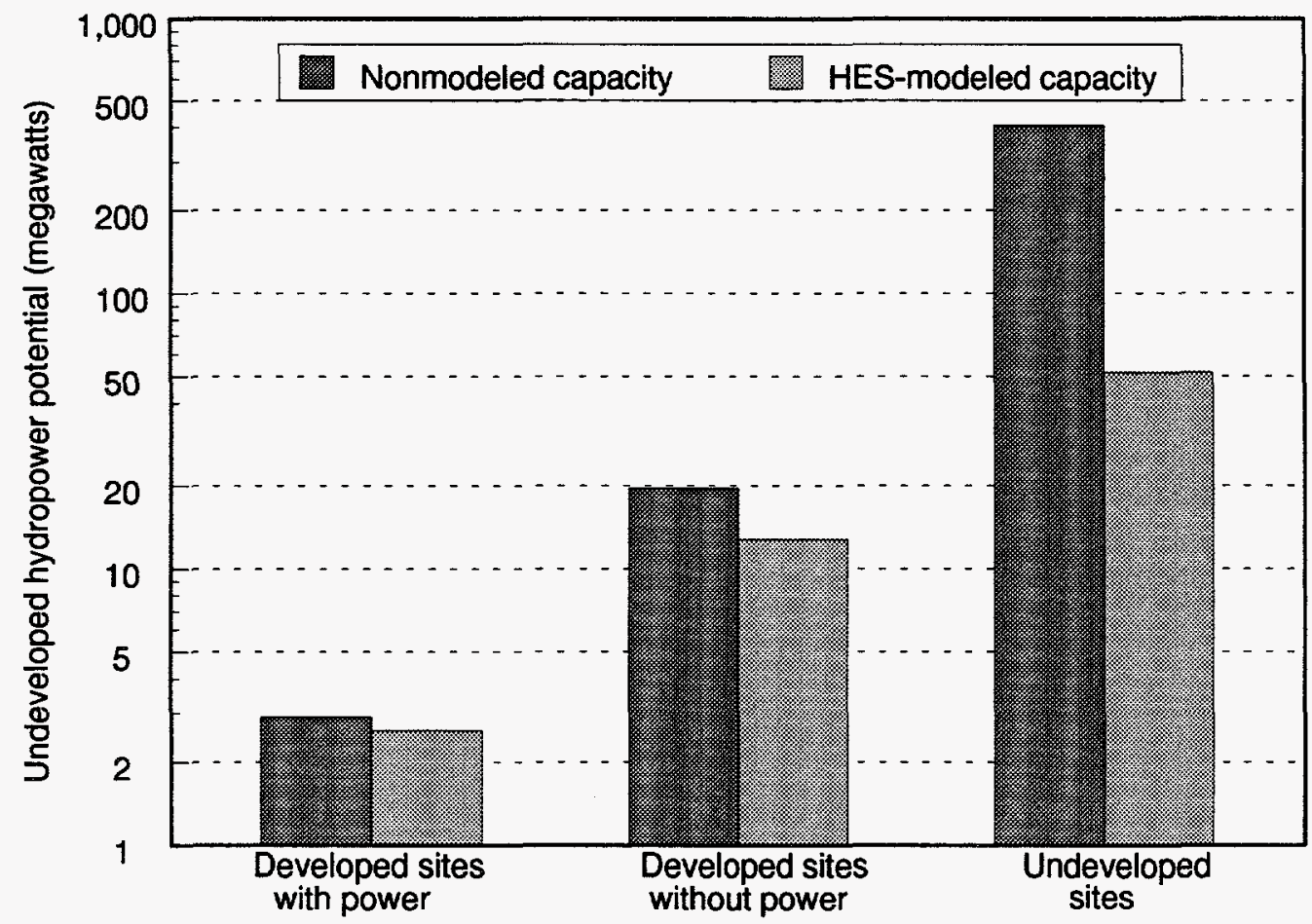

Figure 2. The nonmodeled and HES-modeled undeveloped hydropower potential. 


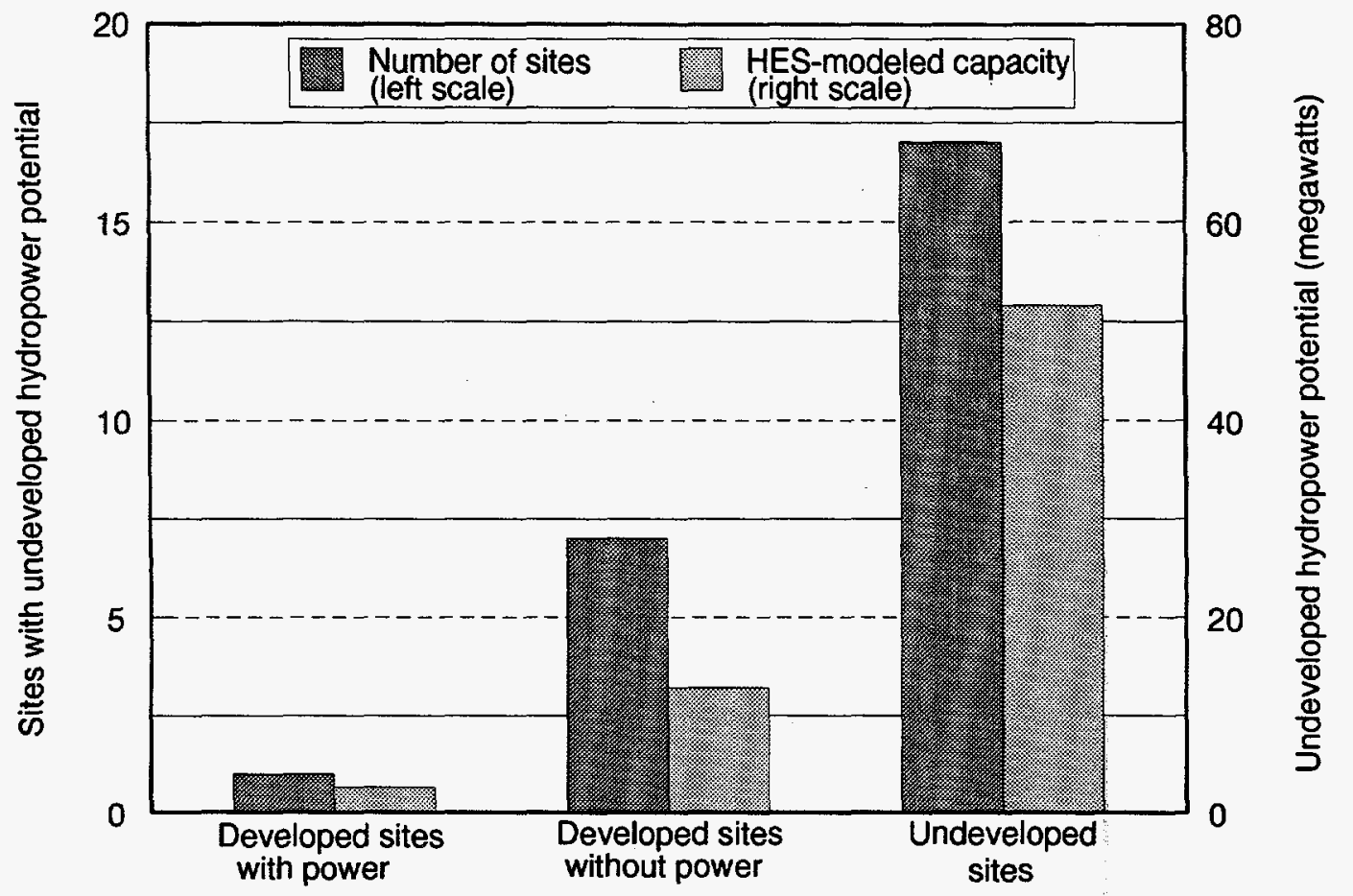

Figure 3. The number of sites with undeveloped hydropower potential and the total megawatts of HESmodeled undeveloped hydropower potential.

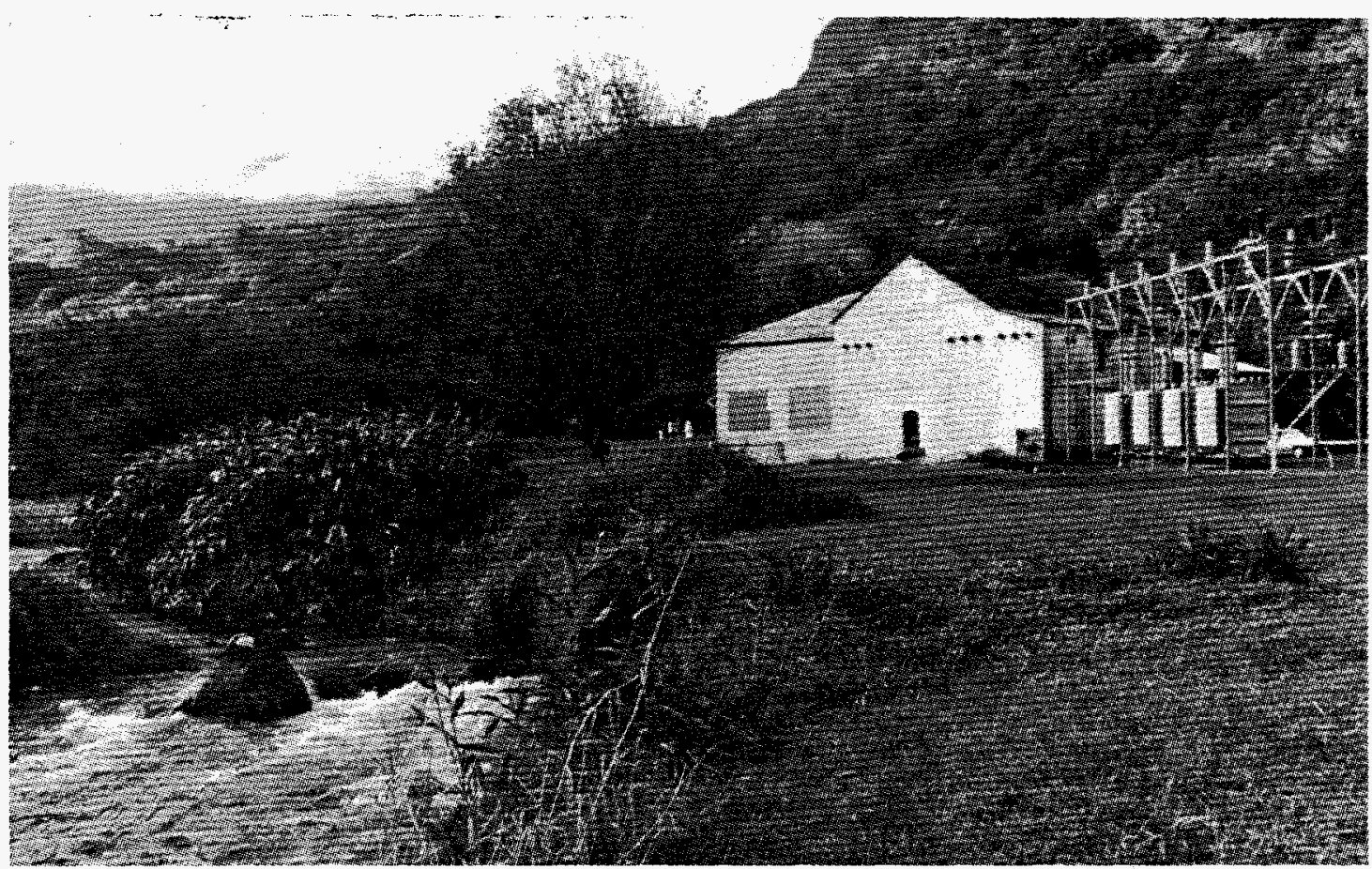

Figure 4. The Wainiha Hydroelectric Plant, on the Island of Kauai, is an example of a developed site in Hawaii. The Wainiha plant, located on the Wainiha River, has a nameplate capacity of $3,600 \mathrm{~kW}$ and it produces an average of 24 million kWh of electricity annually. The plant has a gross hudraulic head of 560 feet and it has an average inflow of about 80 cubic feet per second of water. 


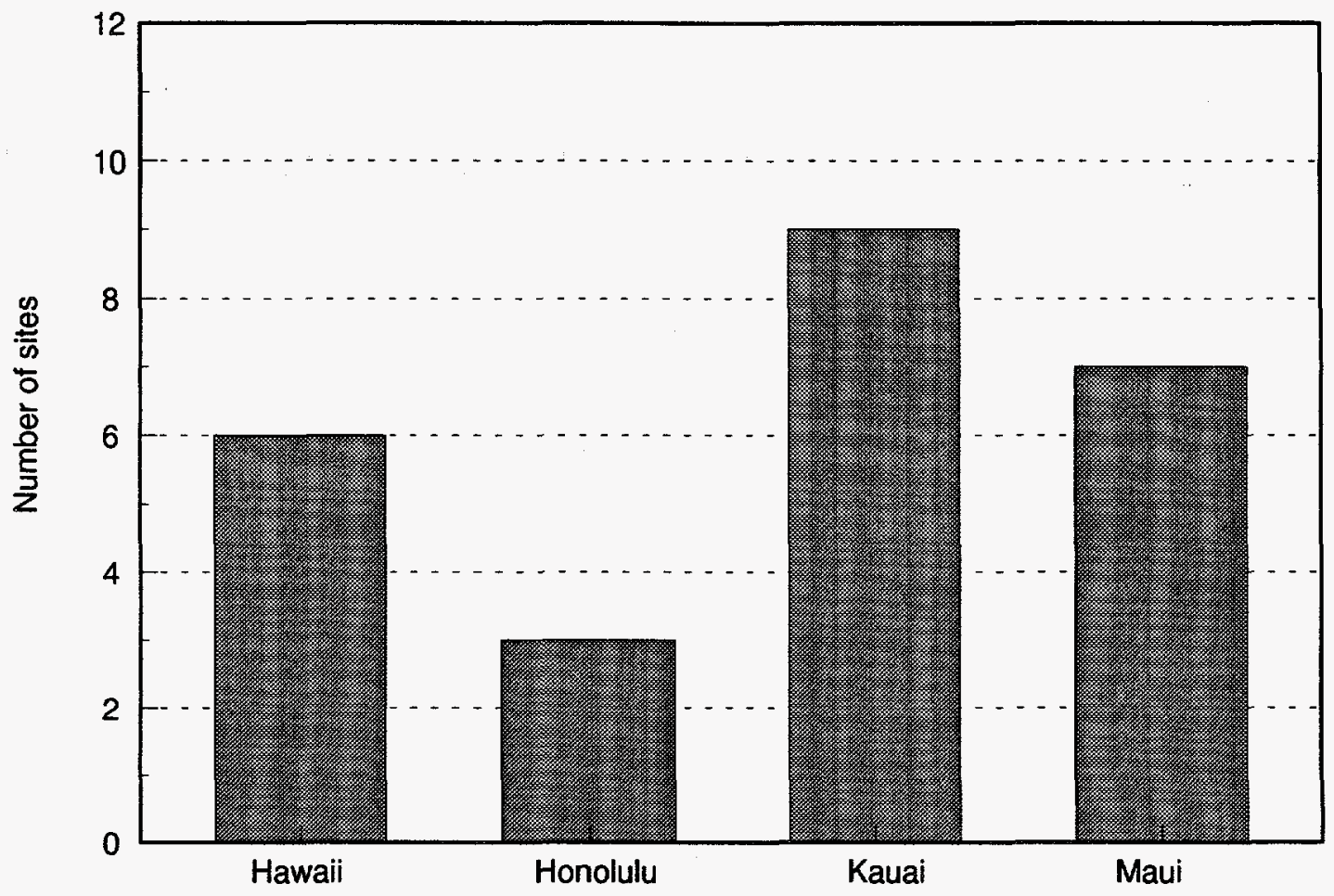

Figure 5. Number of sites with undeveloped hydropower potential in the Hawaii Islands.

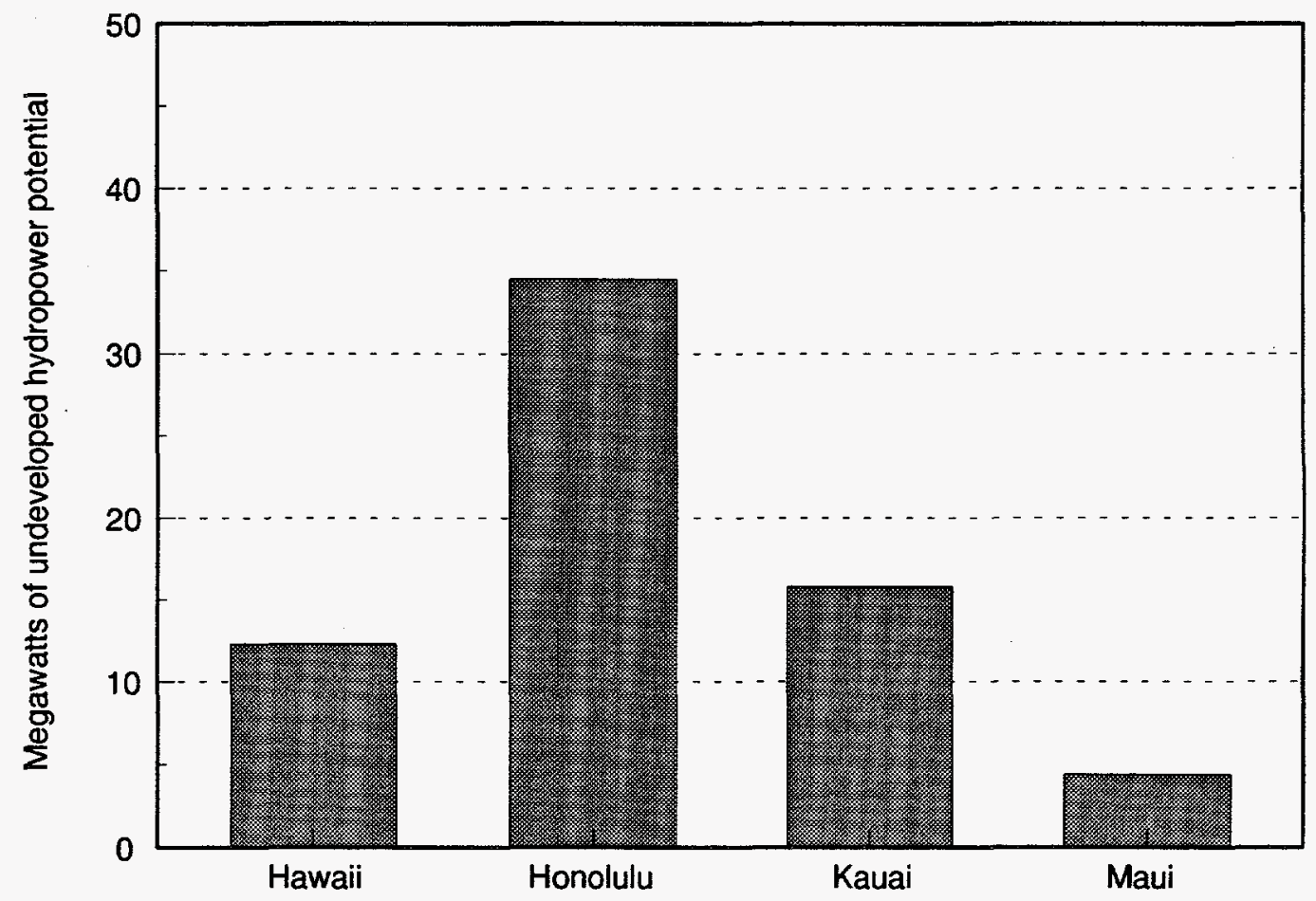

Figure 6. Megawatts of HES-modeled undeveloped hydropower potential in the Hawaii Islands. 
The State of Hawaii also provided information on an additional 19 hydropower sites, having about $10 \mathrm{MW}$ of undeveloped capacity. There is not sufficient data available to model these sites. The 19 sites are included in Appendix D, so the reader is aware that these sites have been identified as potentially having some hydropower capacity.

\section{Detailed Results}

The appendices contain, in the form of HESgenerated reports, detailed information about the undeveloped hydropower potential in Hawaii.

Appendix A summarizes the undeveloped hydropower potential by dam status groups. It provides the number of sites, nonmodeled undeveloped hydropower potential, and HES-modeled undeveloped hydropower potential based on the dam status.

Appendix B lists the project numbers, plant name, stream name, if a site is Federally owned, nonmodeled undeveloped hydropower potential, and HES-modeled undeveloped hydropower potential. The sites are grouped by dam status.

Appendix $\mathbf{C}$ contains a resource database list for each of the 25 sites in Hawaii. Information includes plant name, stream, state, county, river basin and owner names, project number, name plate and HES-modeled undeveloped hydropower potential, the unit and plant types, dam status, latitude, longitude, and the environmental factors that the HES uses to determine the project environmental suitability factor.

Appendix D lists an additional 19 hydropower sites reported by the State of Hawaii. These sites are not included in the HES results.

\section{OBTAINING INDIVIDUAL STATE INFORMATION}

Additional copies of the hydropower resource assessment results for individual states are available and can be obtained by writing or calling the authors or the National Technical Information Service (NTIS).
Telephone Orders-(703) 487-4650. NTIS sales desk and customer services are available between 8:30 a.m. and 5:00 p.m., Eastern Standard Time.

Fax-(703) 321-8547. Customers may fax their orders to NTIS. These orders may be charged to a NTIS deposit account, American Express, VISA, or MasterCard.

Mail Orders-Mail orders should be sent to National Technical Information Service, Document Sales, 5285 Port Royal Road, Springfield, VA 22161. Call the sales desk for prices before placing an order.

Method of Payment-Customers may pay for reports (and other NTIS products and services) by (a) credit card (American Express, Visa or MasterCard); (b) check or money order on a United States bank payable to NTIS; (c) a NTIS deposit account; or, (d) by asking to be billed (add $\$ 7.50$ per order), United States, Canada, and Mexico, only.

Handling Fee-A $\$ 3.00$ handling fee per total order applies to orders from the United States, Canada, and Mexico. Handling charges do not apply to rush order service or pick-up orders.

Postage and Shipping-Orders are shipped first class mail, or equivalent, to addresses in the United States, Canada, and Mexico.

Order Turnaround Time-Orders for technical reports generally are shipped within 2 to 8 days of receipt. For faster service, NTIS offers rush order service.

Rush Order Service-Call 1-800-533-NTIS. In Virginia, Canada, and Mexico call (703) 487-4700. For NTIS rush order service add $\$ 15.00$ per item. This guarantees that an order will be processed through NTIS within 24 hours of its receipt. These orders receive immediate, individual attention. The items ordered are delivered by first call mail. Call NTIS for information on rush order service for computer products.

For Help in Tracing an Order-Call (703) 487-4650 and request the customer service option. 


\section{ADDITIONAL HYDROPOWER EVALUATION SOFTWARE INFORMATION}

Additional information concerning the HES can be obtained by contacting Ben Rinehart or Jim Francfort at the addresses provided below. Copies of the software and the User's Manual may also be obtained from these individuals.

Ben Rinehart, Project Manager

Idaho National Engineering Laboratory

P.O. Box 1625, MS 3830

Idaho Falls, ID 83415-3830

(208) 526-1002
Jim Francfort

Idaho National Engineering Laboratory

P.O. Box 1625, MS 3875

Idaho Falls, ID 83415-3875

(208) 526-6787

Information concerning the State of Hawaii's involvement with the resource assessment or about the identified sites may be obtained by contacting:

David Rezachek

State of Hawaii

P.O. Box 2359

Honolulu, Hawaii 96804-2359

(808) $587-3814$ 


\section{REFERENCES}

Francfort, J. E., S. D. Matthews, and B. N. Rinehart, 1991, Hydropower Evaluation Software User's Manual, DOE/ID-10338, Idaho National Engineering Laboratory, Idaho Falls, Idaho.

Conner, A. M., J. E. Francfort, and B. N. Rinehart, 1996, Uniform Criteria for U.S. Hydropower Resource Assessment, Hydropower Evaluation Software Status Report-1I, DOE/ID 10430.1, Idaho National Engineering Laboratory, Idaho Falls, Idaho. 
Appendix A

\section{Summary Report}




\begin{tabular}{|c|c|c|c|c|}
\hline state & Category & $\begin{array}{l}\text { Number of } \\
\text { Projects }\end{array}$ & $\begin{array}{l}\text { Name Plate } \\
\text { Capacity (KW) }\end{array}$ & $\begin{array}{l}\text { HES Adjusted } \\
\text { Capacity (KW) }\end{array}$ \\
\hline \multirow[t]{4}{*}{$\mathrm{HI}$} & With Power & 1 & 2900 & 2610 \\
\hline & W/O Power & 7 & 19600 & 12840 \\
\hline & Undeveloped & 17 & 405600 & 51571 \\
\hline & STATE TOTAL & 25 & 428100 & 67021 \\
\hline \multirow[t]{3}{*}{ TOTALS } & With Power & 1 & 2900 & 2610 \\
\hline & W/O Power & 7 & 19600 & 12840 \\
\hline & Undeveloped & 17 & 405600 & 51571 \\
\hline \multicolumn{2}{|c|}{ GRAND TOTAL } & 25 & 428100 & 67021 \\
\hline
\end{tabular}




\section{Appendix B}

\section{Hawaii Sites List}


HYDROPOWER RESOURCE ASSESSMENT BY FERC NUMBER

Plant Name/ stream

** FERC Number 10715

UPPER WAILUA

MAHAE ST, N FK WAILUA $R$

** Subtotal **

** FERC Number HIOO2

HONOLII

HONOLII STREAM

** Subtotal **

** FERC Number HI005

HOOPOI CHUTE

WAIHEE DITCH (WAIHEE R)

** Subtotal **

** FERC Number HI007

KAPAIA

HANAMAULU STR

** Subtotal **

** FERC Number HI008

KAUAI

WAINIHA $R$

** Subtotal **

** FERC Number HIOOg

KUALAPUU

KALUA PEEL

** Subtotal **

** FERC Number HI010

KITANO HYDRO

HAELEELE STR/KOKEE DITCH

$\begin{array}{lll}\text { Dam } & \text { Name Plate } & \\ \text { ST Stat } & \text { Rating (KW) PESF } & \text { PESF * KW }\end{array}$

HI U

$1260.00 \quad 0.90$

1134.00

1260.00

1134.00

HI U

$14600.00 \quad 0.10$

1460.00

14600.00

1460.00

HI U

$2000.00 \quad 0.90$

1800.00

2000.00

1800.00

HI U

$120.00 \quad 0.90$

108.00

120.00

108.00

HI U

$25000.00 \quad 0.10$

2500.00

25000.00

2500.00

HI U

$90.00 \quad 0.90$

81.00

90.00

81.00

HI U

$1500.00 \quad 0.90$

1350.00 
Plant Name/ stream

** Subtotal **

** FERC Number HI011

KOKEE WATER PROJECT

KAWAIKOI STR/KOKEE DITCH

** Subtotal **

** FERC Number HI013

WAHIAWA RESERVOIR

KAUKONAHUA STR

** Subtotal **

** FERC Number HI014

WAIHEE

WAIHEE RIVER

** subtotal **

** FERC Number HI016

WAILOA

WAILOA STR

** subtotal **

** FERC Number HI018

WAILUA IKI

EAST \& WEST WAILUA IKI

** Subtotal **

** FERC Number HI02O

UMAUMA

UMAUMA STREAM

** Subtotal ** $\begin{array}{ll}\text { Dam } & \text { Name Plate } \\ \text { ST Stat } & \text { Rating (KW) PESF }\end{array}$

$$
1500.00
$$

1350.00

HI U

$10000.00 \quad 0.10$

1000.00

10000.00

1000.00

HI U

$2800.00 \quad 0.90$

2520.00

2800.00

2520.00

HI U

$730.00 \quad 0.10$

73.00

$$
730.00
$$

73.00

HI U

$2900.00 \quad 0.90$

2610.00

2900.00

2610.00

HI U

$2700.00 \quad 0.25$

675.00

2700.00

675.00

HI U

13800.00

0.25

3450.00

13800.00

3450.00 
HYDROPOWER RESOURCE ASSESSMENT BY FERC NUMBER

$$
\begin{gathered}
\text { Plant Name/ } \\
\text { Stream }
\end{gathered}
$$

** FERC Number HI021

HALAWA

HALAWA STREAM

** Subtotal **

** FERC Number HI024

KOKO CRATER PUMPED HYDRO

PACIFIC OCEAN (AFTERBAY)

** Subtotal **

** FERC Number HI025

KAAU CRATER/MAUNAWILI PH

SEVERAL

** Subtotal **

** FERC Number HI026

NORTH

LUMAHAI RIVER

** Subtotal **

** FERC Number HI019

WAIMEA

WAIMEA $R$

** Subtotal **

** FERC Number 10479

SOUTH FORK WAILUA RIVER

$S$ FK WAILUA $R$

** Subtotal **

** FERC Number 11161

HANALEI

HANALEI $R$

$\begin{array}{lll}\text { Dam } & \text { Name Plate } & \\ \text { ST Stat } & \text { Rating (KW) PESF } & \text { PESF * KW }\end{array}$

HI U

$2100.00 \quad 0.10$

210.00

2100.00

210.00

HI U

$160000.00 \quad 0.10$

16000.00

160000.00

16000.00

HI U

$160000.00 \quad 0.10$

16000.00

160000.00

16000.00

HI U

$6000.00 \quad 0.10$

600.00

6000.00

600.00

HI W

$2900.00 \quad 0.90$

2610.00

2900.00

2610.00

HI WO

$6600.00 \quad 0.90$

5940.00

6600.00

5940.00

HI WO

6000.00

0.10

600.00 
Plant Name/ stream

** Subtotal **

** FERC Number HI003

HONOLII (GARRATT)

HONOLII STREAM

** Subtotal **

** FERC Number HI004

HONOLII (GARRATT)

HONOLII STREAM

** Subtotal **

** FERC Number HI012

UNION MILI

KOHALA DITCH

** Subtotal **

** FERC Number HI022

KUALAPUU RESERVOIR PUMP HY

MOLOKAI IRRIGATION SYSTEM

** Subtotal **

** FERC Number HI023

MOLOKAI INLINE HYDRO

COUNTY WATER LINE

** Subtotal **

*** Total *** $\begin{array}{lll}\text { Dam } & \text { Name Plate } & \\ \text { ST Stat Rating (KW) PESF PESF * KW }\end{array}$

HI Wo

6000.00

600.00

$2400.00 \quad 0.90$

2160.00

2400.00

2160.00

HI Wo

$2400.00 \quad 0.90$

2160.00

2400.00

2160.00

HI Wo

$500.00 \quad 0.90$

450.00

500.00

450.00

HI WO

$1600.00 \quad 0.90$

1440.00

1600.00

1440.00

HI Wo

$100.00 \quad 0.90$

90.00

100.00

90.00

428100.00

67021.00 


\section{Appendix C}

\section{Individual Resource Database List}


DATE : $07 / 02 / 96$

PAGE NO: 1

FERC

Number

Plant Name

Stream

State

10479

10479 SOUTH FORK WAILUA RIVER

S FK WAILUA R

Name

County Name

River Basin

KAUAI

HAWAII ISLANDS RIVER BASIN

Class Owner Name

$\mathrm{R}$ ISLAND PWR CO INC

Name Plate

Rating (KW)

PESF

Annual Energy

6600.00

$0.90 \quad 5940.00$

17500.00

HI

PESF Annual

\begin{tabular}{|c|c|c|c|c|c|}
\hline $\begin{array}{l}\text { Unit } \\
\text { Type }\end{array}$ & Plant & Project & Dam & Latitude & 2202.00 \\
\hline Type & Type & status & Status & Longitude & 15923.00 \\
\hline C & ROR & $\mathrm{xX}$ & Wo & & \\
\hline
\end{tabular}

Factor

Exist Prob

Factor

Exist Prob

Wild/Scenic Protection

wild/Scenic Tributary or

0.90 Wildlife Value

Threatened/Endangered Fish

Upstream/Downstream

Threatened/Endangered Wildife

0.90

wild/Scenic Location

0.90 Federal Land Code 103

0.90 Federal Land Code 104

0.90 Federal Land Code 105

0.90 Federal Land Code 106

0.90 Federal Land Code 107

0.90 Federal Land Code 108

0.90

Cultural value

0.90 Federal Land Code 198

0.90

0.90

0.90

Geologic Value

0.90

0.90

0.90

0.90

0.90

other Value

Recreation Value

Scenic Value

0.90 
DATE: $07 / 02 / 96$

FERC

Number

Plant Name

10715 UPPER WAILUA

County Name

KAUAI

Class

P ISLAND PWR CO INC

Name Plate

Rating (KW)

1260.00

PESF

Annual Energy

PESF* KW

Rating (MWh)

7200.00

Latitude 2205.00

Unit

Plant

Type

Type

Project status

C

ROR

$\mathrm{PA}$

Dam

status

Longitude 15928.00
6480
State

Name

HI
PESF Annual

Energy Rating (MWh)
Factor

Wild/scenic Protection

wild/Scenic Tributary or Upstream/Downstream wild/Scenic Location

Cultural Value

Fish Presence Value

Geologic Value

Historic Value

other value

Recreation Value

Scenic Value
Exist Prob

0.90

Wildlife Value

Threatened/Endangered Fish

Threatened/Endangered Wildlife

0.90 Federal Land Code 103

0.90 Federal Land Code 104

0.90 Federal Land Code 105

0.90 Federal Land Code 106

0.90 Federal Land Code 107

0.90 Federal Land Code 108

0.90 Federal Land Code 198 


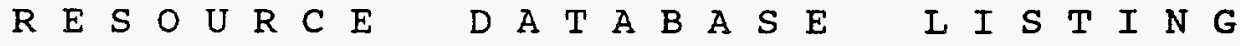

DATE: $07 / 02 / 96$

PAGE NO: 3

FERC

Number

Plant Name

Stream

state

Name

11161 HANALEI

HANALEI R

HI

county Name

KAUAI

Class Owner Name

R HANALEI HYDROPOWER INC

Name Plate

Rating $(\mathrm{KW})$

PESF

Annual Energy

PESF*KW

Rating (MWh)

29000.00

Unit

Plant

Type

Type

C

ROR
Project status

$\mathrm{XX}$
Latitude 2208.00

$\begin{array}{llr}\text { Dam } & \text { Latitude } & 2208.00 \\ \text { Status } & \text { Longitude } & 15928.00\end{array}$

WO
PESF Annual

Energy Rating (MWh)

2900
Factor

Wild/Scenic Protection

wild/Scenic Tributary or Upstream/Downstream wild/Scenic Location Cultural value

Fish Presence Value Geologic Value Historic Value other Value Recreation value Scenic Value
Exist Prob

0.90

Wildife Value

Threatened/Endangered Fish Threatened/Endangered Wildlife

0.90 Federal Land Code 103

$Y \quad 0.75 \quad$ Federal Land Code 104

Y $\quad 0.75$ Federal Land Code 105

0.90

Federal Land Code 106

0.90

Federal Land Code 107

Federal Land code 108

Federal Land Code 198
Exist Prob

Y

0.75

0.90

0.90

0.90

0.90

Y

0.10

0.90

0.90

0.90

0.90
0.90

$\mathrm{Y} \quad 0.90$ 
DATE: $07 / 02 / 96$

FERC

Number

Plant Name

HIOO2 HONOLII

county Name

HAWAII

Class

Owner Name

$R$ MAUNA KEA POWER, INC

Name Plate

Rating (KW)

PESF

PESF*KW

Annual Energy

Rating (MWh)

PESF Annual

14600.00

$0.10 \quad 1460.00$

35000.00

3500

Unit Plant

Type

C

Type

ROR

\section{Project}

status

$\mathrm{XX}$
Factor

Dam
Status

U
State

Name

HI
Factor

Wild/Scenic Protection

Wild/Scenic Tributary or Upstream/ Downstream wild/Scenic Location

Cultural value

Fish Presence Value

Geologic Value

Historic Value other value

Recreation Value

Scenic Value
Exist Prob

0.90

Wildlife Value

Threatened/Endangered Fish Threatened/Endangered Wildlife

0.90 Federal Land Code 103

0.90 Federal Land Code 104

$Y$

0.25

0.90

0.90

$\mathrm{Y} \quad 0.50$

$\mathrm{Y} \quad 0.25$

$\mathrm{Y} \quad 0.50$
Federal Land Code 105

Federal Land Code 106

Federal Land Code 107

Federal Land Code 108

Federal Land Code 198
Exist Prob

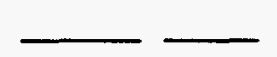

0.25

0.90

0.90

0.90

0.90

0.90

0.90

0.90

0.90

0.90 
DATE : $07 / 02 / 96$

FERC

Number

Plant Name

HI003 HONOLII (GARRATT)

county Name

HAWAII

PAGE NO: 5

State

Name

HI

HONOLII STREAM

Stream

River Basin

HAWAII ISLANDS RIVER BASIN

Class Owner Name

R GARRATT-CALLAHAN CO

Name Plate Rating (KW) PESF

Annual Energy

2400.00

$0.90 \quad 2160.00$

10200.00

PESF Annual

\begin{tabular}{cccccc}
\multicolumn{2}{c}{$2400.00 \quad 0.90$} & 2160.00 & & 10200.00 & 9180 \\
Unit & Plant & Project & Dam & Latitude & 1946.00 \\
Type & Type & Status & Status & Longitude 15511.00 \\
C & ROR & & Wo &
\end{tabular}

Factor

Exist Prob

Factor

Exist Prob

Wild/Scenic Protection

Wild/Scenic Tributary or

0.90 Wildife Value
Threatened/Endan

Threatened/Endangered Fish

Upstream/ Downstream

Threatened/Endangered Wildlife

Exist Prob

0.90 Federal Land Code 103

0.90 Federal Land Code 104

0.90 Federal Land Code 105

0.90 Federal Land Code 106

0.90 Federal Land Code 107

0.90 Federal Land Code 108

0.90

wild/Scenic Location

Cultural Value

Fish Presence Value

0.90

Federal Land Code 198

0.90

0.90

0.90

0.90

0.90

0.90

0.90

0.90

Recreation value

0.90

0.90 
DATE: $07 / 02 / 96$

PAGE NO: 6

FERC

Number

Plant Name

Stream

State

Name

HIOO4 HONOLII (GARRATT)

HONOLII STREAM

HI

county Name

HAWAII
River Basin

HAWAII ISLANDS RIVER BASIN

\section{Class Owner Name}

R GARRATT-CALAHAN CO

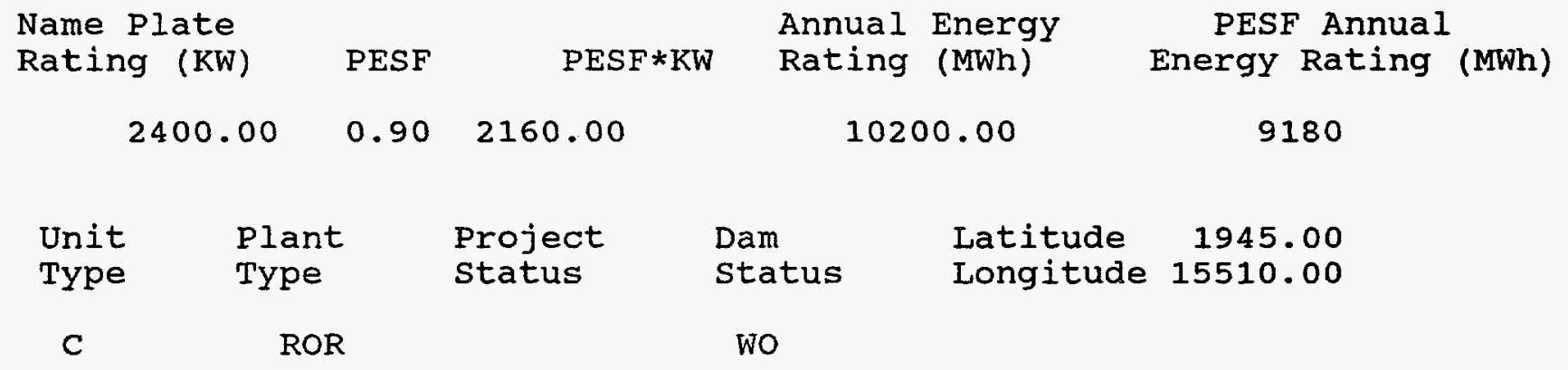

Factor

Wild/Scenic Protection

wild/Scenic Tributary or Upstream/Downstream wild/Scenic Location Cultural value Fish Presence Value Geologic Value Historic Value other value Recreation Value Scenic Value
Exist Prob

0.90

0.90

0.90

0.90

0.90

0.90

0.90

0.90

0.90
Wildiife Value Threatened/Endangered Wildlife

Factor

Exist Prob

Federal Land Code 103

Federal Land Code 104

Federal Land Code 105

Federal Land Code 106

Federal Land Code 107

Federal Land Code 108

Federal Land Code 198
0.90

0.90

0.90

0.90

0.90

0.90

0.90

0.90

0.90

0.90 
R E S O U R E D A T A B A S E I I S T I N G

DATE: $07 / 02 / 96$

PAGE NO: 7

FERC

Number

Plant Name

stream

State

HI005 HOOPOI CHUTE

WAIHEE DITCH(WAIHEE R)

Name

County Name

River Basin

MAUI

HAWAII ISLANDS RIVER BASIN

Class Owner Name

I HAWAIIAN COM \& SUG CO

Name Plate Rating (KW)

Annual Energy

2000.00

PESF Rating (MWh)

PESF Annual

Unit

Plant

$0.90 \quad 1800.00$

3000.00

Energy Rating (MWh)

Type

Type

Project

Status

Dam
status

Latitude 2053.00

C

DIV

$\mathrm{XX}$

$\mathrm{U}$

Factor

Exist Prob

Factor

Exist Prob

Wild/Scenic Protection

wild/Scenic Tributary or

Upstream/Downstream

wild/Scenic Location

Cultural Value

Fish Presence Value

-

Geologic Value

Historic Value

other value

Recreation value

Scenic Value

0.90 Wildlife Value

Threatened/Endangered Fish

Threatened/Endangered Wildlife

0.90 Federal Land Code 103

0.90 Federal Land Code 104

0.90 Federal Land Code 105

0.90 Federal Land Code 106

0.90 Federal Land Code 107

0.90 Federal Land Code 108

0.90 Federal Land Code 198

0.90
HI 
DATE: $07 / 02 / 96$

FERC

Number

Plant Name

HI007 KAPAIA

County Name

KAUAI
PAGE NO: 8

State

Name

HI

HANAMAULU STR

River Basin

HAWAII ISLANDS RIVER BASIN

\author{
Class Owner Name \\ I LIHUE PLANTATION CO
}

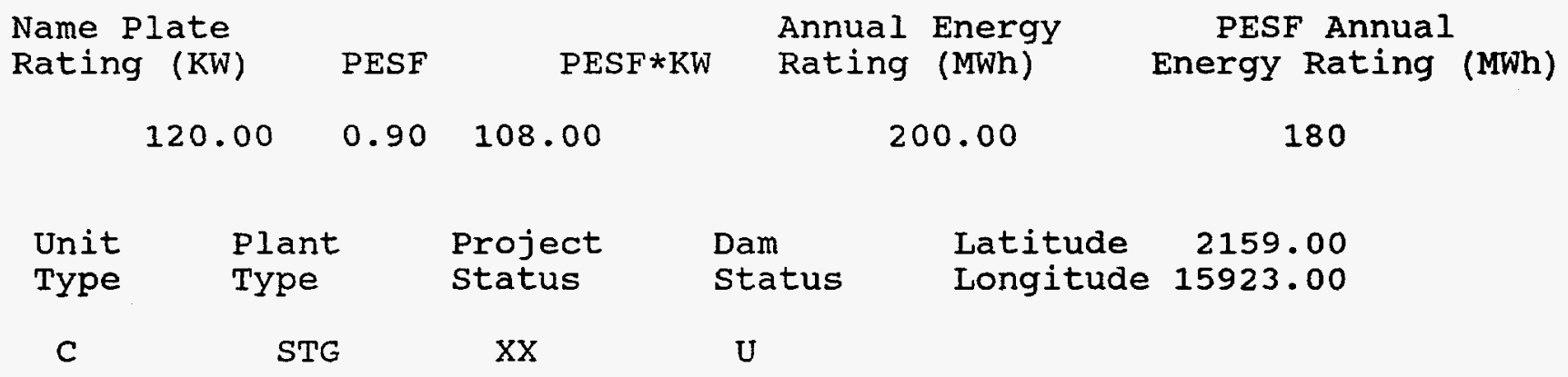

Factor

Exist Prob

Factor

Exist Prob

Wild/Scenic Protection

wild/Scenic Tributary or

0.90 Wildlife Value Threatened/Endangered Fish

0.90

Upstream/Downstream

wild/Scenic Location

Threatened/Endangered Wildlife

0.90

0.90 Federal Land Code 103

0.90

0.90 Federal Land Code 104

0.90

0.90 Federal Land code 105

0.90

Fish Presence Value

0.90 Federal Land Code 106

0.90

Geologic Value

0.90 Federal Land Code 107

0.90

Historic Value

0.90 Federal Land Code 108

0.90

other value

0.90 Federal Land Code 198

0.90

Recreation Value

0.90

0.90 


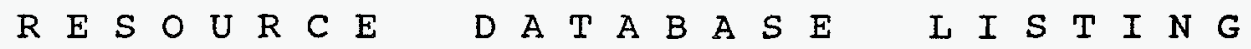

DATE : $07 / 02 / 96$

PAGE NO: 9

FERC

Number

Plant Name

Stream

State

HI008 KAUAI

WAINIHA $R$

Name

County Name

River Basin

KAUAI

HAWAII ISLANDS RIVER BASIN

Class Owner Name

M HAWAII, STATE OF

\begin{tabular}{|c|c|c|c|c|}
\hline $\begin{array}{l}\text { Name Plate } \\
\text { Rating ( } \mathrm{KW})\end{array}$ & PESF & $\mathrm{PESF} * \mathrm{KW}$ & $\begin{array}{l}\text { Annual Energy } \\
\text { Rating (MWh) }\end{array}$ & $\begin{array}{l}\text { PESF Annual } \\
\text { Energy Rating (MWh) }\end{array}$ \\
\hline 25000.00 & 0.10 & 2500.00 & 200000.00 & 20000 \\
\hline
\end{tabular}

Unit Plant Project Dam Latitude 2209.00

Type Type Status Status Longitude 15933.00

C STG $\quad \mathrm{XX} \quad \mathrm{U}$

Factor

Exist Prob

Factor

Exist Prob

Wild/Scenic Protection

Wild/Scenic Tributary or Upstream/Downstream

wild/Scenic Location

cultural value

Fish Presence Value

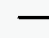

Geologic Value

Historic Value

other value

Recreation value

Scenic Value

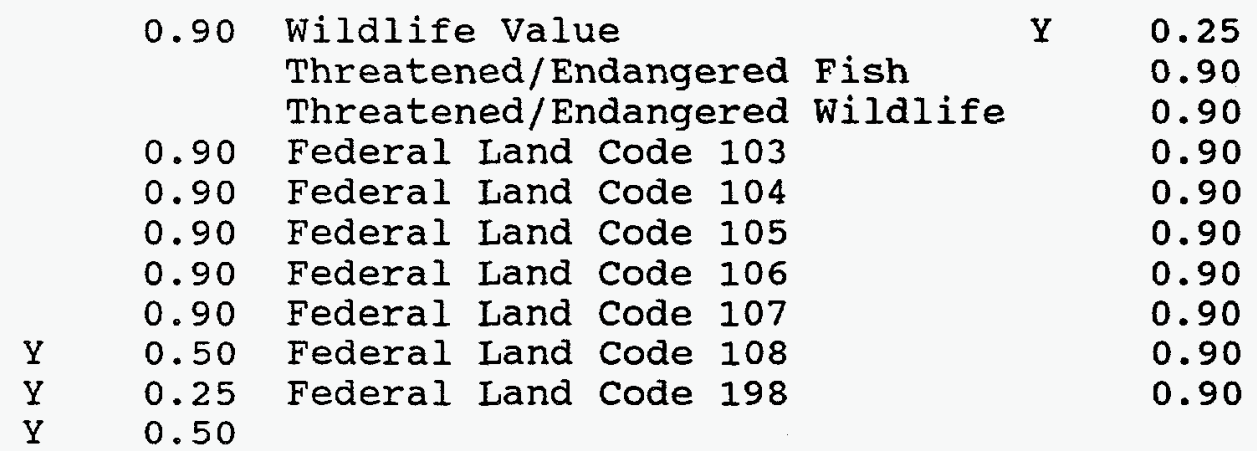


DATE: $07 / 02 / 96$

PAGE NO: 10

FERC

Number

Plant Name

stream

State

HI009 KUALAPUU

KALUA PEEL

Name

county Name

River Basin

MAUI

HAWAII ISLANDS RIVER BASIN

$\begin{array}{cr}\text { Class } & \text { Owner Name } \\ \text { M } & \text { HAWAII, STATE OF }\end{array}$

Name Plate

Rating (KW)

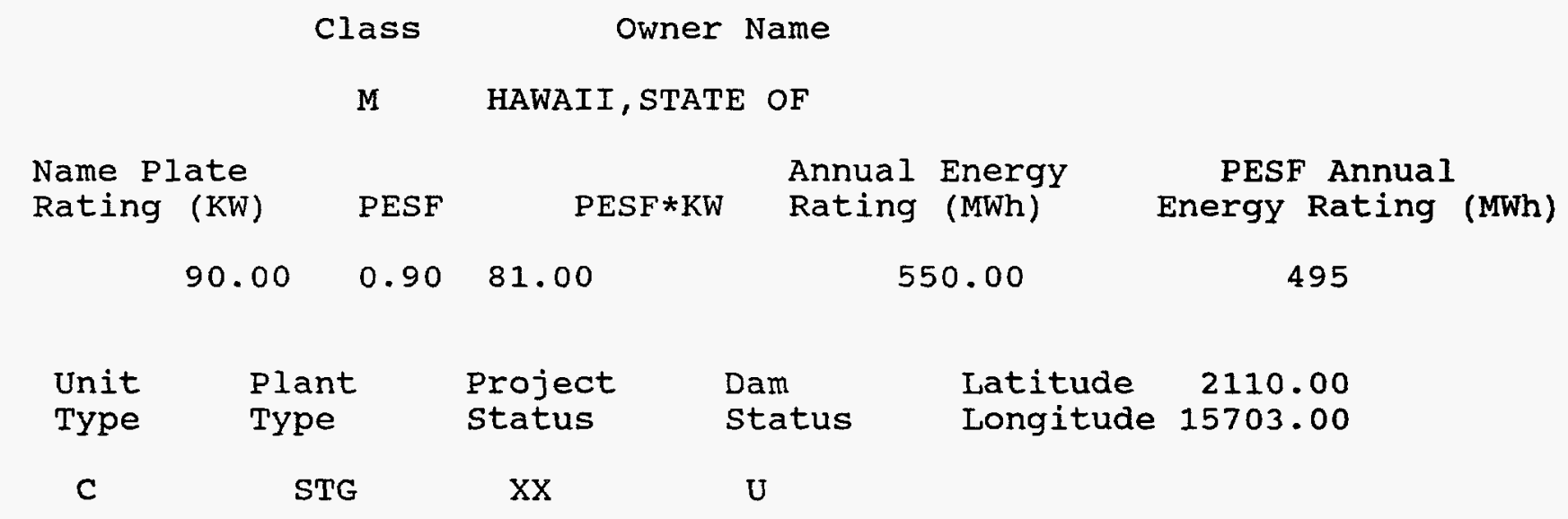

Annual Energy

90.00

$0.90 \quad 81.00$

550.00

HI

Factor

Wild/Scenic Protection

Wild/Scenic Tributary or Upstream/Downstream wild/Scenic Location Cultural value

Fish Presence Value Geologic Value Historic Value other Value

Recreation value

scenic Value
Exist Prob

0.90

Wildlife Value

Threatened/Endangered Fish

Threatened/Endangered Wildlife

0.90 Federal Land Code 103

0.90 Federal Land Code 104

0.90 Federal Land Code 105

0.90 Federal Land Code 106

0.90 Federal Land Code 107

0.90 Federal Land Code 108

0.90 Federal Land Code 198

0.90

Exist Prob

0.90

0.90

0.90

0.90

0.90

0.90

0.90

0.90

0.90

0.90 


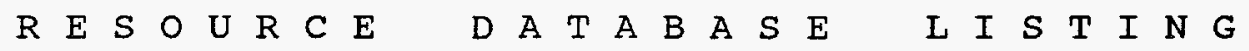

DATE : $07 / 02 / 96$

PAGE NO: 11

FERC

Number

Plant Name

HI010 KITANO HYDRO

HAELEELE STR/KOKEE DITCH

State

Name

County Name

River Basin

KAUAI

HAWAII ISLANDS RIVER BASIN

Class Owner Name

I KEKAHA SUGAR CO LTD

Name Plate

Annual Energy

Rating (KW)

PESF

Rating (MWh)

PESF Annual

1500.00

$0.90 \quad 1350.00$

7600.00

Energy Rating (MWh)

$\begin{array}{cc} & 1500.00 \\ \text { Unit } & \text { Plant } \\ \text { Type } & \text { Type } \\ \text { C } & \text { DIV }\end{array}$

\begin{abstract}
Project
status
\end{abstract}

$\mathrm{xx}$

Exist Prob

Factor

0.90

Wild/Scenic Protection

wild/Scenic Tributary or

Upstream/Downstream

wild/Scenic Location

Cultural value

Fish Presence Value

Geologic Value

Historic Value

other value

Recreation Value

Scenic Value

\section{Dam \\ Status}

$\mathrm{U}$
HI 
DATE: $07 / 02 / 96$

PAGE NO: 12

FERC

Number

Plant Name

Stream

State

HIO11 KOKEE WATER PROJECT

County Name

KAWAIKOI STR/KOKEE DITCH

Name

HIOI1 KOKEE WATER PROJECT

County Name
KAUAI

KAUAI

River Basin

HAWAII ISLANDS RIVER BASIN

\begin{tabular}{|c|c|c|c|c|c|}
\hline \multicolumn{2}{|c|}{ Class } & \multicolumn{2}{|c|}{ Owner Name } & & \\
\hline & M & \multicolumn{2}{|c|}{ TO BE DETERMINED } & & \\
\hline $\begin{array}{l}\text { Name Plate } \\
\text { Rating (KW) }\end{array}$ & PESF & $\mathrm{PESF} * \mathrm{KW}$ & $\begin{array}{l}\text { Annual } \\
\text { Rating }\end{array}$ & $\begin{array}{l}\text { Energy } \\
\text { (MWh) }\end{array}$ & $\begin{array}{l}\text { PESF Annual } \\
\text { Energy Rating (MWh) }\end{array}$ \\
\hline 10000.00 & 0.10 & 1000.00 & \multicolumn{2}{|c|}{29200.00} & 2920 \\
\hline $\begin{array}{l}\text { Unit } \\
\text { Type }\end{array}$ & $\begin{array}{l}\text { Plant } \\
\text { Type }\end{array}$ & $\begin{array}{l}\text { Project } \\
\text { status }\end{array}$ & $\begin{array}{l}\text { Dam } \\
\text { Status }\end{array}$ & \multirow[t]{2}{*}{$\begin{array}{l}\text { Latitude } \\
\text { Longitude }\end{array}$} & $\begin{array}{r}2208.00 \\
15937.00\end{array}$ \\
\hline C & STG & $x x$ & U & & \\
\hline
\end{tabular}

Factor

Exist Prob

Factor

Exist Prob

Wild/Scenic Protection

wild/Scenic Tributary or

0.90 Wildlife Value

$Y$

0.25

Threatened/Endangered Fish

0.90

Upstream/Downstream Threatened/Endangered Wildlife

0.90

wild/Scenic Location

Cultural value

0.90 Federal Land Code 103

0.90

0.90 Federal Land Code 104

0.90

Y 0.25 Federal Land Code 105

0.90

Geologic Value

0.90 Federal Land Code 106

0.90

Historic Value

0.90

Federal Land Code 107

0.90

other Value

Recreation value

Scenic Value

$\begin{array}{ll}\mathrm{Y} & 0.50 \\ \mathrm{Y} & 0.25 \\ \mathrm{Y} & 0.50\end{array}$

Federal Land Code 108

0.90

0.90 
R E S O U R C E D A T A B A S E I I S T I N G

DATE: $07 / 02 / 96$

PAGE NO: 13

FERC

Number

Plant Name

stream

state

HI012 UNION MILL

KOHALA DITCH

Name

County Name

River Basin

HAWAII

HAWAII ISLANDS RIVER BASIN

Class Owner Name

TO BE DETERMINED

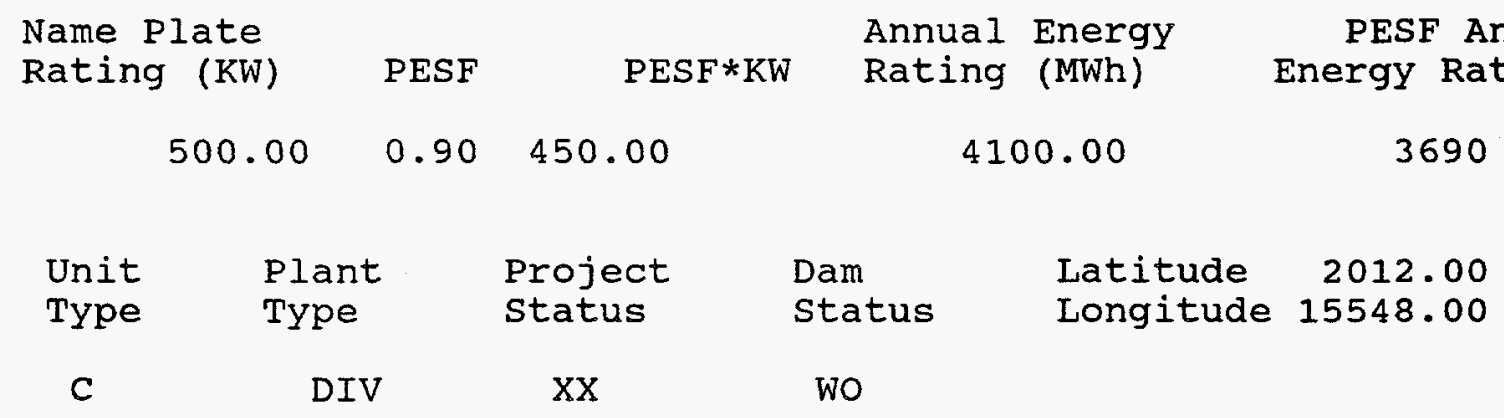

Factor

Exist Prob

Factor

Exist Prob

Wild/Scenic Protection

Wild/Scenic Tributary or

0.90 Wildlife Value

0.90

Upstream/Downstream

wild/Scenic Location

Threatened/Endangered Fish

0.90

Threatened/Endangered Wildlife

0.90

0.90 Federal Land Code 103

0.90

0.90 Federal Land Code 104

0.90

0.90 Federal Land Code 105

0.90

Fish Presence Value

0.90 Federal Land Code 106

0.90

Histc

0.90

Federal Land Code 107

0.90

other Value

0.90

Federal Land Code 108

0.90

Recreation value

0.90

Federal Land Code 198

0.90

Scenic Value

0.90 
DATE: $07 / 02 / 96$

PAGE NO: 14

FERC

Number Plant Name

stream

State

HI013 WAHIAWA RESERVOIR

KAUKONAHUA STR

Name

County Name

HONOLULU

River Basin

HAWAII ISLANDS RIVER BASIN

Class Owner Name

I WAIALUA SUGAR CO

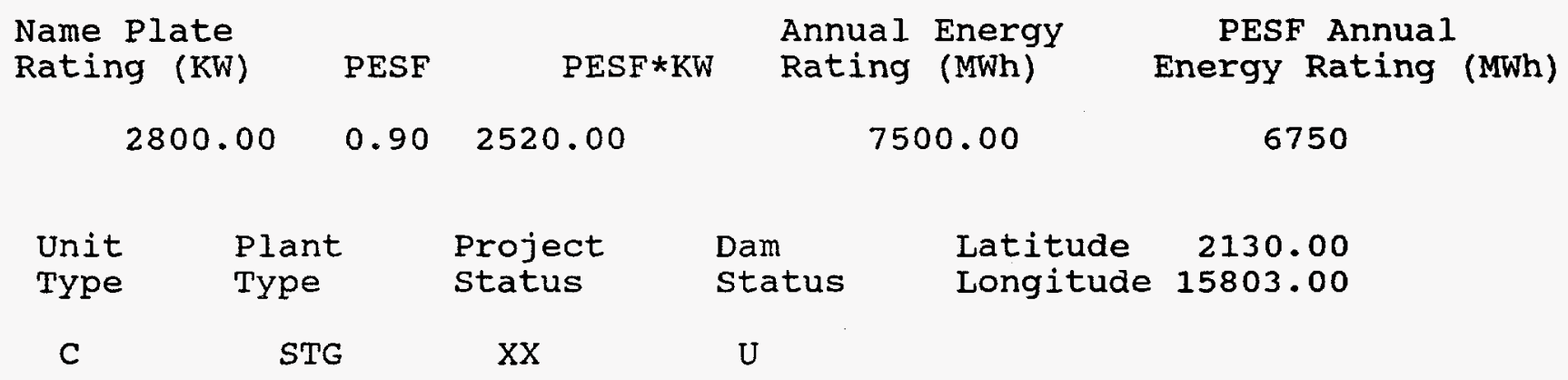

Factor

Exist Prob

Factor

Exist Prob

Wild/scenic Protection

Wild/Scenic Tributary or

0.90 Wildlife Value

Threatened/Endangered Fish

0.90

Threatened/Endangered Wildlife

0.90

Upstream/Downstream

wild/scenic Location

Cultural value

0.90 Federal Land Code 103

0.90

0.90

0.90 Federal Land Code 104

0.90

0.90 Federal Land Code 105

0.90

0.90 Federal Land Code 106

0.90

0.90 Federal Land Code 107

0.90

0.90 Federal Land Code 108

0.90

other value

Recreation Value

0.90

Federal Land Code 198

0.90

Scenic Value

0.90 
R E S O U R C E D A T A B A S E I I S T I N G

DATE: $07 / 02 / 96$

PAGE NO: 15

FERC

Number

Plant Name

stream

State

HIO14 WAIHEE

WAIHEE RIVER

Name

HI014 WAIHEE

county Name

River Basin

MAUI

HAWAII ISLANDS RIVER BASIN

Class Owner Name

TO BE DETERMINED

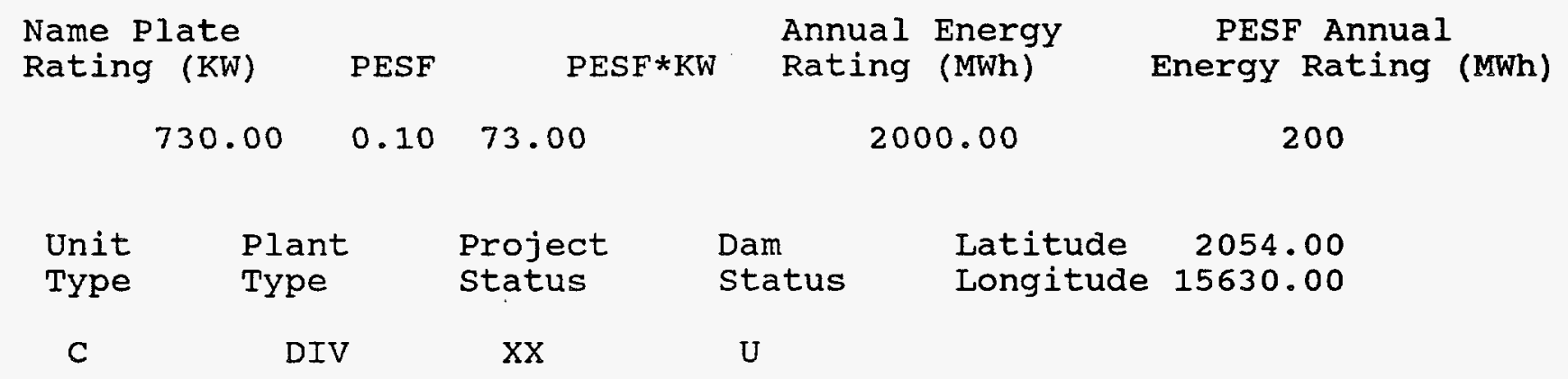

Factor

Exist Prob

Factor

Exist Prob

Wild/Scenic Protection

Wild/Scenic Tributary or

0.90 Wildlife Value Threatened/Endangered Fish

HI

Upstream/Downstream Threatened/Endangered Wildlife

Y

0.25

0.90

Wild/Scenic Location

0.90 Federal Land Code 103

0.90

Cultural Value

0.90 Federal Land code 104

0.90

Fish Presence Value

0.25 Federal Land code 105

0.90

$Y$

0.90 Federal Land Code 106

0.90

Geologic Value

0.90 Federal Land Code 107

0.90

Historic Value

0.90

Other Value

$\begin{array}{ll}Y & 0.50 \\ Y & 0.25\end{array}$

Federal Land Code 108

0.90

Scenic Value

$\mathrm{Y} \quad 0.50$

0.90 
DATE: $07 / 02 / 96$

FERC

Number

HI016 WAILOA

County Name

HAWAII
Plant Name

WAILOA STR

River Basin

HAWAII ISLANDS RIVER BASIN state

Name

HI

\section{Class Owner Name}

TO BE DETERMINED

Name Plate Rating ( $\mathrm{KW})$

2900.00

\section{PESF}

0.90

2610.00
Annual Energy Rating (MWh)

12300.00
PESF Annual Energy Rating (MWh)

\begin{tabular}{|c|c|c|c|c|}
\hline $\begin{array}{l}\text { Unit } \\
\text { Type }\end{array}$ & $\begin{array}{l}\text { Plant } \\
\text { Type }\end{array}$ & $\begin{array}{l}\text { Project } \\
\text { status }\end{array}$ & $\begin{array}{l}\text { Dam } \\
\text { Status }\end{array}$ & $\begin{array}{lr}\text { Latitude } & 2005.00 \\
\text { Longitude } & 15537.00\end{array}$ \\
\hline C & DIV & $\mathrm{XX}$ & $U$ & \\
\hline
\end{tabular}

Factor

Exist Prob

0.90

Wild/Scenic Protection Wild/Scenic Tributary or Upstream/Downstream wild/Scenic Location Cultural Value Fish Presence Value Geologic Value Historic Value other Value Recreation value Scenic Value

0.90

\section{Factor}

Exist Prob

$\begin{array}{ll}0.90 & \text { Wildlife Value } \\ & \text { Threatened/Endangered Fish } \\ & \text { Threatened/Endangered Wildlife } \\ 0.90 & \text { Federal Land Code } 103 \\ 0.90 & \text { Federal Land Code } 104 \\ 0.90 & \text { Federal Land Code } 105 \\ 0.90 & \text { Federal Land Code } 106 \\ 0.90 & \text { Federal Land Code } 107 \\ 0.90 & \text { Federal Land Code } 108 \\ 0.90 & \text { Federal Land Code } 198\end{array}$

0.90

0.90

0.90

0.90

0.90

0.90

0.90

0.90

0.90

0.90 
R E S O U R C E D A T A B A S E I I S T I N G

DATE: $07 / 02 / 96$

PAGE NO: 17

FERC

Number

Plant Name

Stream

State

HI018 WAILUA IKI

EAST \& WEST WAILUA IKI

Name

county Name

River Basin

MAUI

HAWAII ISLANDS RIVER BASIN

Class Owner Name

R GARRATT-CALIAHAN CO

Name Plate

PESF

Annual Energy

PESF*KW Rating (MWh)

PESF Annual

2700.00

0.25675 .00

7900.00

$\begin{array}{lllll}\text { Unit } & \text { Plant } & \text { Project } & \text { Dam } & \text { Latitude } 2048.00 \\ \text { Type } & \text { Type } & \text { Status } & \text { Status } & \text { Longitude } 15609.00\end{array}$

$\begin{array}{llll}\text { C } & \text { ROR } & \mathrm{XX}\end{array}$

Factor

Exist Prob

Factor

Exist Prob

Wild/Scenic Protection

Wild/Scenic Tributary or

Upstream/Downstream

Wild/Scenic Location

Cultural Value

Fish Presence Value

Geologic Value

Historic Value

other value

Recreation Value

Scenic Value

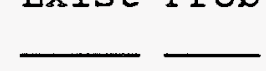

0.90

Wildlife Value

Threatened/Endangered Fish

Threatened/Endangered Wildlife

0.90 Federal Land code 103

0.90 Federal Land Code 104

0.90 Federal Land Code 105

0.90 Federal Land Code 106

0.90 Federal Land Code 107

Y 0.50 Federal Land code 108

0.90 Federal Land Code 198
$\mathrm{HI}$
1975

\section{Energy Rating (MWh)}


DATE : $07 / 02 / 96$

FERC

Number

Plant Name

HI019 WATMEA

county Name

KAUAI

HAWAII ISLANDS RIVER BASIN
State

Name

HI

\section{Class Owner Name}

I KEKAHA SUGAR CO LTD

Name Plate

Rating ( $\mathrm{KW}$ )

2900.00

PESF

0.90

2610.00

Project

status

Type

Plant
Type

C

DIV
Annual Energy

Rating (MWh)

3300.00
PESF Annual Energy Rating (MWh)
Factor

Wild/Scenic Protection wild/Scenic Tributary or Upstream/Downstream wild/Scenic Location Cultural value

Fish Presence Value Geologic Value Historic Value other value Recreation value Scenic Value
Exist Prob

0.90

Wildlife Value

Threatened/Endangered Fish

Threatened/Endangered Wildlife

0.90 Federal Land Code 103

0.90 Federal Land Code 104

0.90 Federal Land Code 105

0.90 Federal Land Code 106

0.90 Federal Land Code 107

0.90 Federal Land Code 108

0.90 Federal Land Code 198
Exist Prob

0.90

0.90

0.90

0.90

0.90

0.90

0.90

0.90

0.90

0.90 


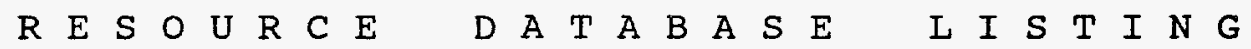

DATE : $07 / 02 / 96$

PAGE NO: 19

FERC

Number

Plant Name

stream

State

HI020 UMAUMA

UMAUMA STREAM

Name

County Name

River Basin

HAWAII

HAWAII ISLANDS RIVER BASIN

Class Owner Name

TO BE DETERMINED

Name Plate

$\begin{array}{lll} & \text { Annual Energy } \\ \text { PESF } & \text { PESF*KW } & \text { Rating (MWh) }\end{array}$

PESF Annual

Rating ( $\mathrm{KW}$ )

0.253450 .00

40200.00

Energy Rating (MWh)

13800.00

0.25

Unit Plant

Project

Dam

Status

Latitude 1953.00

Type

Type

status

C

ROR

$\mathrm{XX}$

$\mathrm{U}$

Factor

Wild/Scenic Protection

Wild/Scenic Tributary or

Upstream/Downstream

wild/Scenic Location

Cultural value

Fish Presence Value

Geologic Value

Historic Value

other Value

Recreation value

Scenic Value
Exist Prob

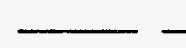

0.90

Wildife Value Threatened/Endangered Fish Threatened/Endangered Wildlife

0.90 Federal Land Code 103

0.90 Federal Land Code 104

0.90 Federal Land Code 105

0.90 Federal Land Code 106

0.90 Federal Land Code 107

$\mathrm{Y} \quad 0.50$

0.90

Federal Land Code 108

Federal Land Code 198
HI
10050

0.90

Exist Prob

Y

0.25

0.90

0.90

0.90

0.90

0.90

0.90

0.90

0.90

0.90 
FERC

Number

HIO2 1

HAIAAWA

Plant Name

county Name

MAUI

Class

Owner Name

TO

EETERMINED

Name Plate Rating ( $\mathrm{KW})$

\section{PESF}

Annual Energy

2100.00

0.10

210.00
Project status

$\mathrm{XX}$
Stream

HALAWA STREAM

River Basin

HAWAII ISLANDS RIVER BASIN state

Name

HI

$\begin{array}{ccclc}\text { Unit } & \text { Plant } & \text { Project } & \text { Dam } & \text { Latitude } 2109.00 \\ \text { Type } & \text { Type } & \text { Status } & \text { Status } & \text { Longitude 15646.00 } \\ \text { C } & \text { ROR } & \text { XX } & U & \end{array}$

Factor

Exist Prob

0.90

Wild/Scenic Protection Wild/Scenic Tributary or Upstream/Downstream wild/Scenic Location Cultural value Fish Presence Value Geologic Value Historic Value other value Recreation Value Scenic Value
Factor

$\begin{array}{ccc} & 0.90 & \begin{array}{l}\text { Wildlife Value } \\ \text { Threatened/Endangered }\end{array} \\ & & \text { Threatened/Endangered } \\ & 0.90 & \text { Federal Land Code } 103 \\ & 0.90 & \text { Federal Land Code } 104 \\ & 0.90 & \text { Federal Land Code } 105 \\ & 0.90 & \text { Federal Land Code } 106 \\ & 0.90 & \text { Federal Land Code } 107 \\ \text { Y } & 0.50 & \text { Federal Land Code } 108 \\ Y & 0.25 & \text { Federal Land Code } 198 \\ Y & 0.50 & \end{array}$

PESF Annual Energy Rating (MWh)

990
Exist Prob

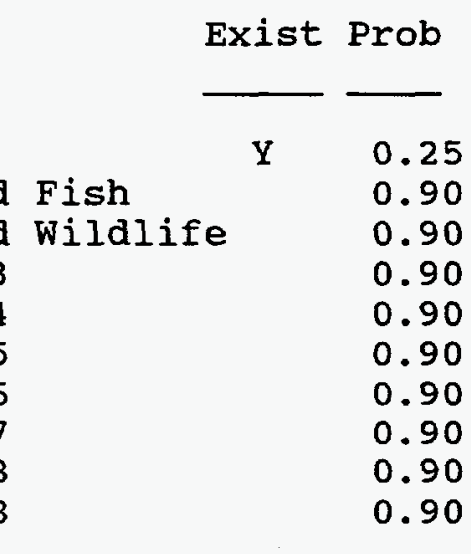

0.90

0.90

. 90

0.90

0.90

0.90 
R E S O U R C E D A T A B A S E I I S T I N G

DATE: $07 / 02 / 96$

PAGE NO: 21

FERC

Number

Plant Name

stream

state

HIO 022

KUALAPUU RESERVOIR PUMP

HY MOLOKAI

IRRIGATION SYSTEM

County Name

MAUI

River Basin

HAWAII ISLANDS RIVER BASIN

HI

Name

Class Owner Name

TO BE DETERMINED

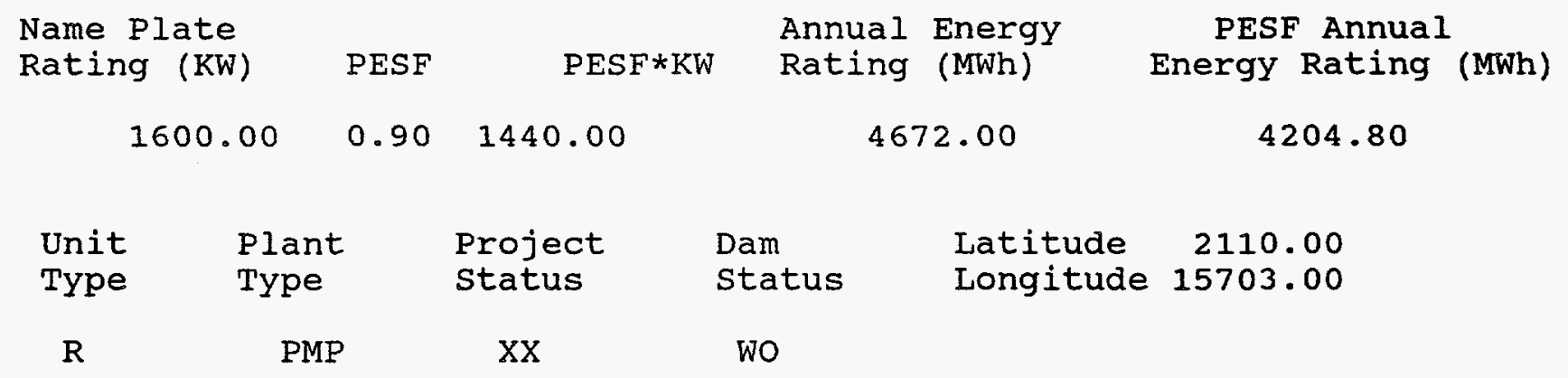

Factor

Wild/Scenic Protection

Wild/Scenic Tributary or Upstream/Downstream wild/Scenic Location Cultural Value Fish Presence Value Geologic Value Historic Value other Value Recreation Value Scenic Value
Exist Prob

0.90

Wildlife Value Threatened/Endangered Fish Threatened/Endangered Wildlife

0.90 Federal Land Code 103

0.90 Federal Iand Code 104

0.90 Federal Land Code 105

0.90 Federal Land Code 106

0.90 Federal Land Code 107

0.90 Federal Land Code 108

0.90

0.90
Exist Prob

0.90

0.90

0.90

0.90

0.90

0.90

0.90

0.90

0.90

0.90 
FERC

Number

Plant Name

HI023 MOLOKAI INLINE HYDRO

county Name

MAUI stream

COUNTY WATER LINE

River Basin

HAWAII ISLANDS RIVER BASIN state

Name

HI

\section{Class Owner Name}

P UTILITY/COUNTY

Name Plate

Rating (KW)

100.00
PESF

$0.90 \quad 90.00$

Project status

Type

$$
\begin{aligned}
& \text { Plant } \\
& \text { Type } \\
& \text { ROR }
\end{aligned}
$$

C
Annual Energy Rating (MWh)

200.00
PESF Annual Energy Rating (MWh) 180
Factor

Wild/Scenic Protection Wild/Scenic Tributary or Upstream/Downstream wild/Scenic Location Cultural value Fish Presence Value Geologic Value Historic Value other value Recreation Value Scenic Value
Exist Prob

0.90 Wildife Value Threatened/Endangered Fish Threatened/Endangered Wildlife

0.90 Federal Land Code 103

0.90 Federal Land Code 104

0.90 Federal Land Code 105

0.90 Federal Land Code 106

0.90 Federal Land Code 107

0.90 Federal Land Code 108

0.90 Federal Land Code 198

$$
\text { Latitude } 2109.00
$$

Wo 
R E S O U R C E D A T A B A S E I I S T I N G

DATE: $07 / 02 / 96$

PAGE NO: 23

FERC

Number

Plant Name

Stream

State

HIO 24

KOKO CRATER PUMPED HYDRO

PACIFIC OCEAN (AFTERBAY)

Name

county Name

River Basin

HONOLULU

HAWAII ISLANDS RIVER BASIN

$\begin{array}{cc}\text { Class } & \text { Owner Name } \\ \text { P } & \text { HAWAIIAN ELECTRIC COMPANY, INC }\end{array}$

Name Plate

Rating (KW)

PESF

Annual Energy

PESF Annual

160000.00

$0.10 \quad 16000.00$

Energy Rating (MWh)

HI

Unit

Plant

Project

Type

Type

status

R

PMP

$\mathrm{XX}$

Factor

Exist Prob

Wild/Scenic Protection

wild/scenic Tributary or

Upstream/Downstream

Wild/Scenic Location

Cultural value

Fish Presence Value

Geologic Value

Historic Value

other value

Recreation Value

Scenic Value
0.90

Wildlife Value

Threatened/Endangered Fish

Threatened/Endangered Wildlife

0.90 Federal Land Code 103

Y $\quad 0.50$ Federal Land Code 104

0.90

0.90

Federal Land Code 105

Federal Land Code 106

Federal Land Code 107

Federal Land Code 108

$Y \quad 0.50$

0.50

$\begin{array}{ll}\mathrm{Y} & 0.25 \\ \mathrm{Y} & 0.50\end{array}$

Federal Land Code 198
346020.00

Dam

Status

Latitude 2117.00

$\mathrm{U}$

34602
Exist Prob

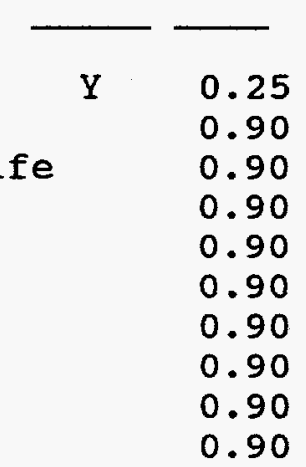


FERC

Number

$\mathrm{HIO} 25$
Plant Name

KAAU CRATER/MAUNAWILI PH

county Name

HONOLULU
Stream

SEVERAL

River Basin

HAWAII ISLANDS RIVER BASIN state

Name

HI

Class Owner Name

P HAWAIIAN ELECTRIC COMPANY, INC

Name Plate Rating (KW)

160000.00
PESF

0.10

16000.00

Plant Type

Type

PMP

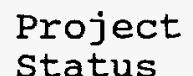

$\mathrm{XX}$
$\mathbf{R}$
Annual Energy

Rating (MWh)

350400.00
PESF Annual

Energy Rating (MWh)
Factor

Wild/Scenic Protection wild/Scenic Tributary or Upstream/Downstream Wild/Scenic Location Cultural Value Fish Presence Value Geologic Value Historic Value other value Recreation Value Scenic Value
Exist Prob

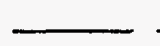

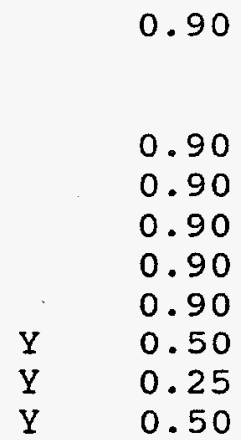

Dam status Latitude 2120.00 Longitude 15747.00

$\mathrm{U}$

Factor

Wildlife Value Threatened/Endangered Fish Threatened/Endangered Wildlife Federal Land code 103 Federal Land code 104 Federal Land code 105 Federal Land Code 106 Federal Land Code 107 Federal Land Code 108 Federal Land Code 198
Exist Prob

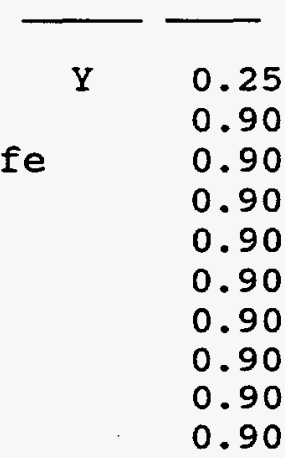




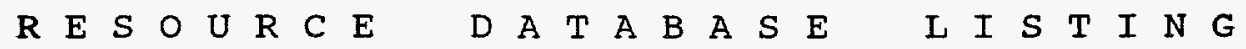

DATE: $07 / 02 / 96$

PAGE NO: 25

FERC

Number

Plant Name

Stream

State

HI026 NORTH

LUMAHAI RIVER

Name

HI

county Name

KAUAI
River Basin

HAWAII ISLANDS RIVER BASIN

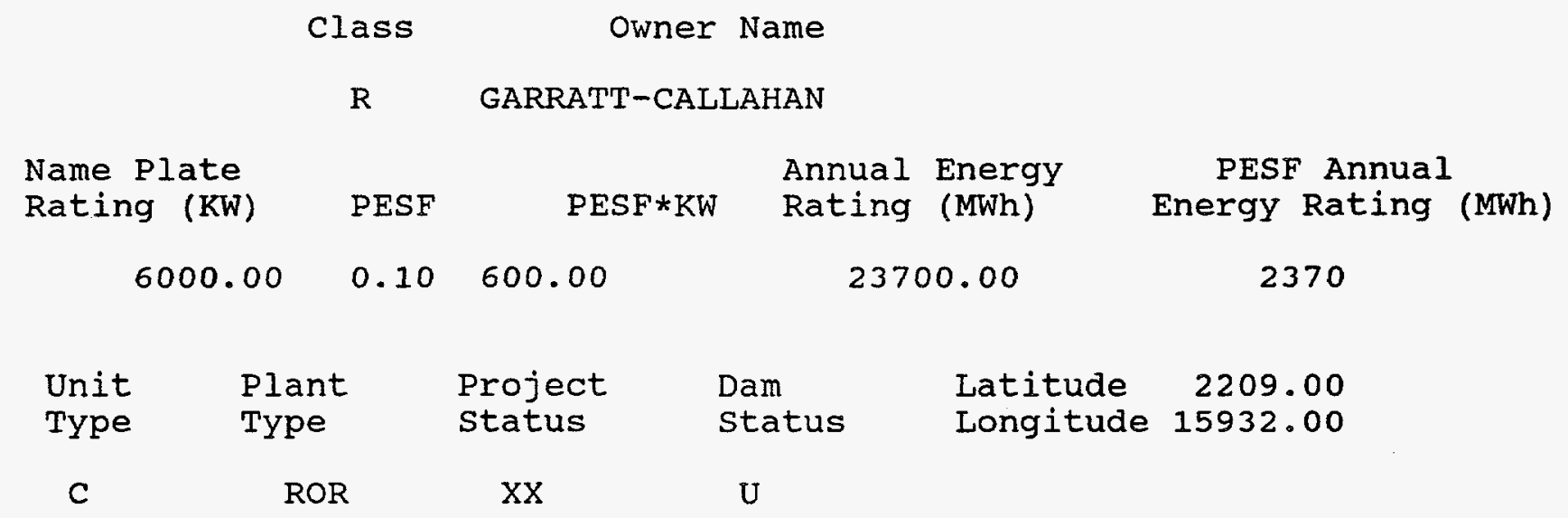

Factor

Wild/Scenic Protection

wild/Scenic Tributary or

Upstream/Downstream

wild/Scenic Location

Cultural value

Fish Presence Value

Geologic Value

Historic Value

other value

Recreation value

Scenic Value
Exist Prob

0.90

Wildlife Value

Threatened/Endangered Fish

Threatened/Endangered Wildlife

0.90 Federal Land Code 103

0.90 Federal Land Code 104

Y $\quad 0.25$ Federal Land Code 105

0.90

0.90

$Y \quad 0.50$

$Y \quad 0.25$

$Y \quad 0.50$
Federal Land Code 106

Federal Land Code 107

Federal Land Code 108

Federal Land Code 198
Exist Prob

Y

0.25

0.90

0.90

0.90

0.90

0.90

0.90

0.90

0.90

0.90 


\section{Appendix D \\ List of 19 Additional Hydropower Sites}

D-1 


\begin{tabular}{|c|c|c|c|c|c|}
\hline $\begin{array}{c}\text { Location/Name } \\
\text { of Project }\end{array}$ & Stream & $\begin{array}{l}\text { Estimated } \\
\text { Capacity } \\
(\mathrm{kW})\end{array}$ & $\begin{array}{c}\text { Estimated } \\
\text { Generation } \\
(\text { million } \\
\mathrm{kWh} / \mathrm{yr})\end{array}$ & Owner & County \\
\hline & Alia Stream & 330 & 1.5 & & Hawaii \\
\hline & Awini Falls & 1,500 & 7.7 & & Hawaii \\
\hline \multirow[t]{2}{*}{ Hakalau Mill } & Hakalau/Kolekole & 75 & $n / a$ & Hilo Coast Processing Company & Hawaii \\
\hline & Honokane Nui Stream & 1,100 & 6.2 & & Hawaii \\
\hline \multirow[t]{2}{*}{ Papaikou Mill } & Honolii Stream & 130 & 1.0 & Hilo Coast Processing Company & Hawaii \\
\hline & Keaiwa-Meyer Reservoirs & 280 & 1.7 & & Hawaii \\
\hline Hawi & Kohala Ditch & 350 & $\mathrm{n} / \mathrm{a}$ & Kohala Sugar Company & Hawaii \\
\hline Paauhau & Lower Hamakua Ditch & 150 & $\mathrm{n} / \mathrm{a}$ & Paauhau Sugar Company & Hawaii \\
\hline \multirow[t]{8}{*}{ Wainaku Mill } & Maili Stream & 60 & $\mathrm{n} / \mathbf{a}$ & Hilo Coast Processing Company & Hawaii \\
\hline & Pohakupuka Stream & 600 & 2.3 & & Hawaii \\
\hline & Hanawi Stream & 1,000 & 5.0 & & Maui \\
\hline & Honokohau (Honolua) Ditch & 130 & 0.8 & & Maui \\
\hline & Kahakuloa Stream & 230 & 1.6 & & Maui \\
\hline & Kolea & 1,100 & 4.5 & & Maui \\
\hline & Nailiilihaele Stream & 470 & 3.0 & & Maui \\
\hline & Pelekenu Stream & 860 & 3.8 & & Molokai \\
\hline Upper Lihue & North Wailua \& Lliilihua Ditch & 800 & 5.7 & Lihue Plantation Co. & Kauai \\
\hline Lower Lihue & North Wailua \& Lliilihua Ditch & 1,000 & 7.1 & Lihue Plantation Co. & Kauai \\
\hline Malumalu & Waihuhanu Stream & $\underline{128}$ & n/a & McBryde Sugar Company & Kauai \\
\hline & & 10,293 & 51.9 & & \\
\hline
\end{tabular}

